\title{
The Impact of Catechol-O-Methyltransferase and Dopamine D4 Receptor Genotypes on Neurophysiological Markers of Performance Monitoring
}

\author{
Ulrike M. Krämer, ${ }^{1}$ Toni Cunillera, ${ }^{2}$ Estela Càmara, ${ }^{1,3}$ Josep Marco-Pallarés, ${ }^{1}$ David Cucurell, ${ }^{2}$ Wido Nager, ${ }^{1,3}$ \\ Peter Bauer, ${ }^{4}$ Rebecca Schüle, ${ }^{5}$ Ludger Schöls, ${ }^{5}$ Antoni Rodriguez-Fornells, ${ }^{2,6}$ and Thomas F. Münte ${ }^{1}$ \\ ${ }^{1}$ Department of Neuropsychology, Otto-von-Guericke-University, 39106 Magdeburg, Germany, ${ }^{2}$ Department Psicologia Bàsica, University of Barcelona, \\ 08035 Barcelona, Spain, ${ }^{3}$ Medical University of Hannover, 30163 Hannover, Germany, ${ }^{4}$ Medical Genetics, University of Tübingen, 72074 Tübingen, \\ Germany, ${ }^{5}$ Hertie-Institute for Clinical Brain Research, University of Tübingen, 72076 Tübingen, Germany, and ${ }^{6}$ Institució Catalana de Recerca i Estudis \\ Avançats, 08010 Barcelona, Spain
}

Dynamic adaptations of one's behavior by means of performance monitoring are a central function of the human executive system, that underlies considerable interindividual variation. Converging evidence from electrophysiological and neuroimaging studies in both animals and humans hints at the importance of the dopaminergic system for the regulation of performance monitoring. Here, we studied the impact of two polymorphisms affecting dopaminergic functioning in the prefrontal cortex [catechol- $O$-methyltransferase (COMT) Val108/158Met and dopamine D4 receptor (DRD4) single-nucleotide polymorphism (SNP)-521] on neurophysiological correlates of performance monitoring. We applied a modified version of a standard flanker task with an embedded stop-signal task to tap into the different functions involved, particularly error monitoring, conflict detection and inhibitory processes. Participants homozygous for the DRD4 $T$ allele produced an increased error-related negativity after both choice errors and failed inhibitions compared with $C$-homozygotes. This was associated with pronounced compensatory behavior reflected in higher post-error slowing. No group differences were seen in the incompatibility N2, suggesting distinct effects of the DRD4 polymorphism on error monitoring processes. Additionally, participants homozygous for the COMT Val allele, with a thereby diminished prefrontal dopaminergic level, revealed increased prefrontal processing related to inhibitory functions, reflected in the enhanced stop-signal-related components N2 and P3a. The results extend previous findings from mainly behavioral and neuroimaging data on the relationship between dopaminergic genes and executive functions and present possible underlying mechanisms for the previously suggested association between these dopaminergic polymorphisms and psychiatric disorders as schizophrenia or attention deficit hyperactivity disorder.

Key words: executive functions; COMT; DRD4; error-related negativity; inhibition; dopamine

\section{Introduction}

Performance monitoring enables humans to adapt their behavior and comprises error detection and correction, functions that are instigated by the anterior cingulate cortex (ACC), inferior frontal gyrus, dorsolateral prefrontal cortex (PFC), and insular cortex (Carter et al., 1998; Gehring and Knight, 2000; Ullsperger and von Cramon, 2001). Considerable interindividual variability in performance monitoring raises the question for underlying differences in neurotransmitter systems, particularly dopamine functioning (Cohen et al., 2002; Seamans and Yang, 2004).

A role of dopamine in performance monitoring is suggested by altered executive functions in diseases such as schizophrenia

Received Sept. 14, 2007; revised Nov. 10, 2007; accepted Nov. 12, 2007.

This work was supported by a grant from the Volkswagen-Foundation (T.F.M., A.R.F., L.S.). We thank Andrea Seibel for excellent technical assistance in sequencing and genotyping procedures. Special thanks are devoted to Immaculada Clemente for all the provided facilities.

Correspondence should be addressed to Thomas F. Münte, Department of Neuropsychology, 0tto-von-GuerickeUniversity, Universitätsplatz 2,39106 Magdeburg, Germany. E-mail: thomas.muente@med.ovgu.de.

DOI:10.1523/JNEUROSCI.4229-07.2007

Copyright $\odot 2007$ Society for Neuroscience 0270-6474/07/2714190-09\$15.00/0 and Parkinson's disease (Falkenstein et al., 2001; Laurens et al., 2003) and the rich dopaminergic innervation of aforementioned prefrontal areas (Seamans and Yang, 2004). Moreover, a previous theory posits a central role of the mesencephalic dopaminergic system in the generation of the error-related negativity (ERN), a neurophysiological marker of action monitoring that is generated in the ACC (Holroyd and Coles, 2002).

Previous studies hint at genetic differences that may account for interindividual variation in dopaminergic functioning and thereby in performance monitoring (Fossella et al., 2002; Blasi et al., 2005). One frequently studied single-nucleotide polymorphism (SNP) is located in the catechol-O-methyltransferase (COMT) gene and refers to a G-to-A change at codon 158/108, resulting in a Valine to Methionine substitution. COMT is a major enzyme in dopamine degradation particularly in prefrontal areas because of a lack of the dopamine transporter in this region (Chen et al., 2004). The Methionine allele leads to a threefold to fourfold reduction in COMT activity (Chen et al., 2004). This polymorphism has been related to altered performance in tests for executive functions and differences in both prefrontal brain 
Table 1. Demographic, behavioral, and EEG data

\begin{tabular}{|c|c|c|c|c|}
\hline & C/Met & C/Val & T/Met & T/Val \\
\hline $\operatorname{Sex}(F / M)$ & $6 / 4$ & $7 / 3$ & $6 / 4$ & $7 / 3$ \\
\hline Age (mean, years) & 20.3 & 20.2 & 23.8 & 21.4 \\
\hline RT (compatible) & 362.2 & 402.2 & 367.6 & 360.4 \\
\hline RT (incompatible) & 391.6 & 424.8 & 394.4 & 385.9 \\
\hline RT difference & 29.4 & 22.6 & 26.8 & 25.5 \\
\hline RT (go errors) & 303.6 & 342.3 & 319.8 & 309.6 \\
\hline Percentage of go errors & 12.0 & 9.2 & 9.4 & 8.9 \\
\hline Posterror slowing & 13.8 & 15.8 & 11.6 & 12.4 \\
\hline Post-noninhibition slowing & 8.0 & -2.7 & 18.0 & 19.1 \\
\hline Percentage of corrected errors & 80.2 & 88.4 & 89.7 & 86.7 \\
\hline Stop-signal delay & 103.2 & 134.2 & 114.2 & 116.6 \\
\hline SSRT & 271.5 & 275.6 & 263.4 & 249.7 \\
\hline Percentage of artifact rejections & 20.2 & 15.7 & 13.6 & 15.4 \\
\hline Included go errors & 146.5 & 123.8 & 130.6 & 124.8 \\
\hline Included stop errors & 129.9 & 137.3 & 137.9 & 132.9 \\
\hline
\end{tabular}

RT, Reaction time of correct responses in milliseconds; Percentage of go errors, percentage of error trials of all go trials; Percentage of corrected errors, the number of corrected go errors relative to all go errors; Percentage of artifact rejection, the average percentage of rejected EEG epochs caused by artifacts; Included go and stop errors, the average number of included error trials after artifact rejection; M, male; F, female.

activation (Egan et al., 2001) and PFC-midbrain interaction (Akil et al., 2003; Meyer-Lindenberg et al., 2005).

The dopamine D4 receptor (DRD4) gene, which shows preferential expression in the PFC (Oak et al., 2000), has been implicated in prefrontal dysfunctions in schizophrenia and attention deficit hyperactivity disorder (ADHD) (Okuyama et al., 1999; Bellgrove et al., 2005a). The SNP -521 refers to a $C$-to- $T$ substitution, with the $T$ allele resulting in $40 \%$ less transcriptional efficiency (Okuyama et al., 1999; but see Kereszturi et al., 2006).

Here we investigated the combined impact of these two polymorphisms on neurophysiological correlates (event-related potentials, ERPs) of performance monitoring. We used a combined flanker-stop-signal paradigm, which has been widely used to investigate error detection and correction (Gehring et al., 1993; Rodriguez-Fornells et al., 2002) and inhibitory processes (Pliszka et al., 2000; Liotti et al., 2005), and moreover in behavioral studies tapping into effects of dopaminergic polymorphisms (Fossella et al., 2002). Because imaging studies demonstrated higher prefrontal activity related to attentional control in COMT Val homozygotes (Blasi et al., 2005), we expected an enhancement of neurophysiological correlates of performance monitoring as the ERN and N2, that emanate from the ACC and PFC. Previous results regarding the DRD4 polymorphism are only tentative, but given the suggested higher risk for schizophrenia in $C$ homozygotes (Okuyama et al., 1999) (but see Mitsuyasu et al., 2007), we hypothesized rather diminished prefrontal functioning in this group.

\section{Materials and Methods}

All procedures were approved by the local ethical Institutional Review Board (IRB00003099).

Participants. The genotyping was performed in a large sample of 656 students from the University of Barcelona (491 women; age range from 18 to 56 ; mean, 21.7; SD, 3.5), who underwent a comprehensive neuropsychological test battery. We initially performed the genotyping for six different polymorphisms in the dopaminergic system, namely COMT Val108/158Met, DRD4-521, DRD4 120 bp, DRD4 exon III, monoamine oxidase A (MAO-A) $30 \mathrm{bp}$, and dopamine transporter (DAT1) variable number of tandem repeat polymorphism. The allele frequencies for the different polymorphisms were as follows: COMT methionine, $45.7 \%$; DRD4-521 T allele, 52.9\%; DRD4 120 bp 2-repeat, 78.5\%. The DRD4 exon III allele frequencies were as follows: $8.7 \%$ (2 repeat), $2.7 \%$ (3 repeat), 70.9 (4 repeat), 1.5\% (5 repeat), $0.3 \%$ (6 repeat), and $15.9 \%$ (7 repeat). The MAO-A 30 bp allele frequencies were $0.3 \%$ ( 2 repeat), $30.9 \%$
(3 repeat), 1.8\% (3.5 repeat), $65.9 \%$ (4 repeat) and $1.2 \%$ (5 repeat). DAT1 allele frequencies were $0.2 \%$ ( 8 repeat), $35.4 \%$ ( 9 repeat), $64.4 \%$ (10 repeat) and $0.1 \%$ (11 repeat).

Genotype effects on parameters in the neuropsychological test battery were most promising for the COMT and DRD4-521 polymorphisms (these results will be presented elsewhere). We thus selected 53 (36 women; age range, $18-34$ years; mean, 21.2) participants based on their DRD4-521 and COMT alleles for the ERP-session. All were righthanded participants of European ancestry, except one Peruvian-Spanish man, and were free of neurological and psychiatric disorders (self report). They were paid for their participation and gave written informed consent. We included only participants who were homozygous for both polymorphisms, yielding a two-by-two factorial design with the four groups T-Val, T-Met, C-Val, and C-Met. Two participants had to be excluded because of a genotyping error (TC-Met instead of TT-Met and TC-Val instead of TT-Val) and one (T-Met) did not follow the instructions (no inhibitions in stop trials) (see below). We thus had 10 participants in the T-Met group and excluded respectively participants in the other groups based on their data quality (percentage of artifact rejections) to have the same number of participants in each group ( $n=10$; in total $n=40,26$ women; for the gender and age distribution in each group) (Table 1). The allele frequencies in these 40 participants for the other genotyped polymorphisms were as follows: DRD4 exon III, $11.3 \%$ (2 repeat), 1.6\% (3 repeat), $72.6 \%$ (4 repeat), $3.2 \%$ (5 repeat), $11.2 \%$ (7 repeat); DRD4 120 bp 2 repeat, $78.2 \%$; DAT1, 35.9\% (9 repeat), $64.1 \%$ (10 repeat); MAO-A 30 bp, $21.3 \%$ (3 repeat), $5 \%$ (3.5 repeat), $68.7 \%$ (4 repeat) and 5\% (5 repeat). The allele frequencies of the nonstudied polymorphisms are thus comparable in the initial sample and the ERP sample. ${ }^{a}$ It might be, that the other genotyped polymorphisms also have an influence on the processes under study, which we missed with the current selection. Obviously, the other genotyped polymorphisms as well as other polymorphisms of the DRD4 and COMT gene (Barr, 2001; DiazAsper et al., 2006), or other polymorphisms affecting dopamine or other neurotransmitter systems might have an additional impact on neural correlates of performance monitoring.

Genotyping. DNA contributed to the study was prepared by standard techniques from two independent EDTA blood samples of each participant. Genotyping of the $-521 \mathrm{C} / \mathrm{T}$ polymorphism in the DRD4 promoter (Okuyama et al., 1999) as well as the COMT G-to-A polymorphism at codon 108/158 (short/long isoform) resulting in valine to methionine substitution (Lachman et al., 1996) was performed using real-time fluorescence resonance energy transfer PCR. The region spanning the SNP was amplified with the primers DRD4 for $\left(5^{\prime}\right.$ CTG AGG GCC AGA GGC TG 3')/DRD4rev (5' GAG GAT CAA CTG TGC AAC GG $3^{\prime}$ ) and COMT for (5' GGG CCT ACT GTG GCT ACT CA $3^{\prime}$ )/COMTrev (5' TTC AGT GAA CGT GGT GTG AAC A $3^{\prime}$ ), respectively. The polymorphic base nucleotide is covered by the fluorescein labeled donor probe (DRD4sensor 5' CGG GCG TGG AGG GCG CG-Fl 3'; COMTsensor 5' ATT TCG CTG GCA TGA AGG ACA A-Fl 3'). The adjacent acceptor probe (DRD4anchor 5' LCRed610GAC TCG CCT CGA CC-LCRed610-TCG T 3'; COMT anchor 5' LCRed610- GTG TGC ATG CCT GAC CCG TTG TCA-ph 3') was labeled with LCRed640 (Roche, Grenzach-Wyhlen, UK). Melting curve analysis of the matrix-probe duplex is allele-dependent and allows discrimination of the two SNP alleles. Primers and probes were designed and synthesized by Tib Molbiol (Berlin, Germany). Ampli-

${ }^{a}$ Participants for the ERP session were selected randomly from the respective genotype groups in the initial sample, without considering their individual performance in the neuropsychological test battery. They can be thus assumed to be representative for the initial sample. 
fication and melting analysis were performed on a Light Cycler480 automate instrument (Roche). For PCR amplification the LightCycler480 genotyping master (Roche) was used in a 384-well format with $10 \mu \mathrm{l}$ reaction volumes. Cycling conditions with touchdown annealing temperatures from $65^{\circ} \mathrm{C}$ to $55^{\circ} \mathrm{C}$ over the first 10 cycles were as follows: $10 \mathrm{~min}, 95^{\circ} \mathrm{C}, 45$ cycles with $20 \mathrm{~s}$ annealing temperature; $20 \mathrm{~s}, 72^{\circ} \mathrm{C}$; and $20 \mathrm{~s}, 95^{\circ} \mathrm{C}$ followed by a high-resolution melting curve from $50^{\circ} \mathrm{C}$ to $85^{\circ} \mathrm{C}$ with continuous fluorescence acquisition.

Genotypes of participants selected for ERP were controlled in an independent second DNA sample by direct sequencing using the ABI PRISM BigDye Terminator v3.1 Cycle Sequencing Kit (Applied Biosystems, Foster City, CA). Sequencing products were resolved on an ABI 3100 automated Sequencer (Applied Biosystems) and analyzed using the Staden Package (Bonfield et al., 1995).

Paradigm. We applied a modified variant of the Eriksen flanker task (Eriksen and Eriksen, 1974) that required the participants to respond to the central arrow in an array of five arrows (with the right hand after a right-directed arrow and vice versa). The four surrounding arrows were either compatible or incompatible to the central arrow, favoring performance errors. We presented $33.3 \%$ of compatible and $50 \%$ of incompatible trials. In the remaining $16.6 \%$ of trials, we included no-go trials, following a variant of the stop-signal paradigm (Band et al., 2003). In these trials, the central green arrow changed to red after a variable delay, indicating participants to inhibit the response in these trials. The delay was adapted to participants behavior by means of a staircase tracking algorithm (Band and van Boxtel, 1999) as follows. The stop-signal delay was set to $140 \mathrm{~ms}$ initially. After a successful inhibition the stop-signal delay was increased by $10 \mathrm{~ms}$ (making the inhibition harder), after a failure in inhibition the stop-signal delay was reduced by $10 \mathrm{~ms}$ (making the inhibition easier). This procedure was applied to yield an inhibition rate of $\sim 50 \%$. We computed the stop-signal reaction time (Band et al., 2003) by subtracting the participant's mean stop-signal delay from the median reaction time of correct go responses. Each stimulus array was presented in the middle of the screen. Stimulus duration was $300 \mathrm{~ms}$ and the stimulus onset asynchrony was fixed to $900 \mathrm{~ms}$. Participants received 20 training trials to get acquainted to the task. They were encouraged to correct their errors in the go trials as fast as possible. The experiment was divided in eight blocks, each comprising 240 trials, resulting in a total amount of 1920 trials.

ERPs. The electroencephalogram (EEG) was recorded from 29 tin electrodes mounted in an elastic cap (electrode positions: Fp1/2, F3/4, C3/4, P3/4, O1/2, F7/8, T3/4, T5/6, FC1/2, FC5/6, CP1/2, CP5/6, PO1/2, Fz, Cz, $\mathrm{Pz}$ ) with reference electrodes placed on the right and left mastoids. During recording, all scalp electrodes were referenced against an average reference and off-line re-referenced against the algebraic mean of the activity at the two mastoid electrodes. Electrode impedances were kept below $5 \mathrm{k} \Omega$. Vertical eye movements and blinks were monitored by an electrode placed below the right eye. EEG and EOG were recorded continuously and digitized with a sampling rate of $250 \mathrm{~Hz}$ (bandpass from 0.01-70 Hz). After individualized rejection of eye and muscle artifacts, stimulus- and response-locked averages were obtained for the different conditions ( $-100-924 \mathrm{~ms}$ for stimulus-locked averages and $-400-600$ $\mathrm{ms}$ for response-locked ERPs). In the stimulus-locked ERPs, the $100 \mathrm{~ms}$ preceding the stimulus was considered as baseline; in the responselocked ERPs, baseline was defined as the $50 \mathrm{~ms}$ before the button press. Only reaction time responses that were produced between 120 and 750 $\mathrm{ms}$ after the stimulus were considered in the behavioral and ERP analyses. All artifact-free error trials were included regardless of a subsequent corrective response.

For statistical analyses, mean amplitudes (unless otherwise stated) were subjected to repeated measures ANOVA with the betweensubject factors COMT ( Met vs $\mathrm{Val}$ ) and DRD4 ( $T$ vs $C$ ) and the within-subject factors condition and electrode position, dependent on the particular component (as stated below). For all statistical effects involving more than one degree of freedom in the numerator, the Huynh-Feldt correction was applied to correct for possible violations of the sphericity assumption (Huynh and Feldt, 1976). The corrected probabilities together with the corresponding $\varepsilon$-values are reported. Because our approach to genotype effects on the neuro- physiological correlates of performance monitoring was hypothesesdriven (see Introduction), and because we restricted our analysis to previously described ERP components, we did not apply a Bonferroni correction for multiple comparisons.

\section{Results}

\section{Behavioral results}

Participants reacted faster in compatible (mean reaction time $373 \pm 33 \mathrm{~ms}$ SD) than in incompatible $(399 \pm 36)$ go trials, being in line with usual findings in flanker tasks $\left(F_{(1,36)}=121.23, p<\right.$ 0.001 ). The mean percentage of errors was $9.9 \%$ ( \pm 5.9$)$, being higher in incompatible trials $(12.3 \%)$ than in compatible trials $\left(6.2 \% ; F_{(1,36)}=215.57, p<0.001\right)$, and error responses were as expected faster than correct responses (errors, $319 \pm 37 \mathrm{~ms}$; correct responses, $\left.390 \mathrm{~ms} \pm 35.2 ; F_{(1,36)}=311.82, p<0.001\right)$. The mean percentage of inhibited no-go trials was $48.8 \%$ ( \pm 2.6$)$, with an average stop-signal delay of $117 \mathrm{~ms}( \pm 39)$. Participants corrected on average $86.3 \%$ ( \pm 22.9 ) of their errors with a response time of the corrections of $204 \mathrm{~ms}$ (relative to the first response; \pm 59 ). Behavioral data of the four groups are depicted in Table 1. Participants with the $\mathrm{C} / \mathrm{C}$ - $\mathrm{Val} / \mathrm{Val}$ genotype were slower in their overall reaction time in both their correct and their erroneous responses (COMT by DRD4 interaction, $F_{(1,36)}=5.35, p=$ 0.027).

In agreement with previous findings in the flanker task, we observed slower responses in trials after errors compared with trials after correct responses (posterror slowing, $13.4 \pm 16.4 \mathrm{~ms}$ ) (Rabbitt, 1966). The same result was observed for the trials that followed failed inhibitions (post-noninhibition slowing, $10.6 \pm$ $18.9 \mathrm{~ms}$ ). Interestingly, participants with the DRD4 T/T genotype reduced their speed after failed inhibitions significantly more than individuals with the $C / C$ genotype (main effect DRD4, $\left.F_{(1,36)}=8.46, p=0.006 ; T, 18.5 \mathrm{~ms} ; C, 2.6 \mathrm{~ms}\right)$. Moreover, carriers of the $T$ allele had a shorter stop-signal reaction time (SSRT) (Band et al., 2003), reflecting faster inhibitory processes in these participants $\left(F_{(1,36)}=4.85, p=0.034 ; T, 256.6 \mathrm{~ms} ; C\right.$, $273.6 \mathrm{~ms}$ ).

\section{Event-related potentials}

The percentage of EEG epochs that were rejected because of artifacts was $16.2 \%( \pm 7.1)$ with no difference between the groups (main effects and interaction of COMT and DRD $4, p>0.1$ ). The mean number of errors in go trials (reactions with the wrong hand) included in the average after artifact rejection was 131.4 ( \pm 80.6 ), whereas the mean number of false alarms in no-go trials was 134.5 ( \pm 12.9 ). Both did not differ between the groups (all main effects and interactions of COMT and DRD $4, p>0.1$ ). The percentage of artifact rejections and included errors in the four groups are reported in Table 1.

\section{ERPs: response-locked data}

Both errors in go trials (in the following referred to as choice errors) and in no-go trials (referred to as stop errors) led to an increased negativity peaking shortly after the error, resembling the well known ERN component with its characteristic frontocentral distribution (Falkenstein et al., 1990; Gehring et al., 1993) (Figs. 1, 2). The negativity after choice errors peaked at $\sim 60 \mathrm{~ms}$ after the response, whereas the negativity after stop errors peaked slightly later at $\sim 90 \mathrm{~ms}$ after the response. Results for both conditions will be reported separately.

\section{Go trials}

The ERN for choice errors was clearly present but differentially pronounced in the four groups (Fig. 1). First visual inspection 
A

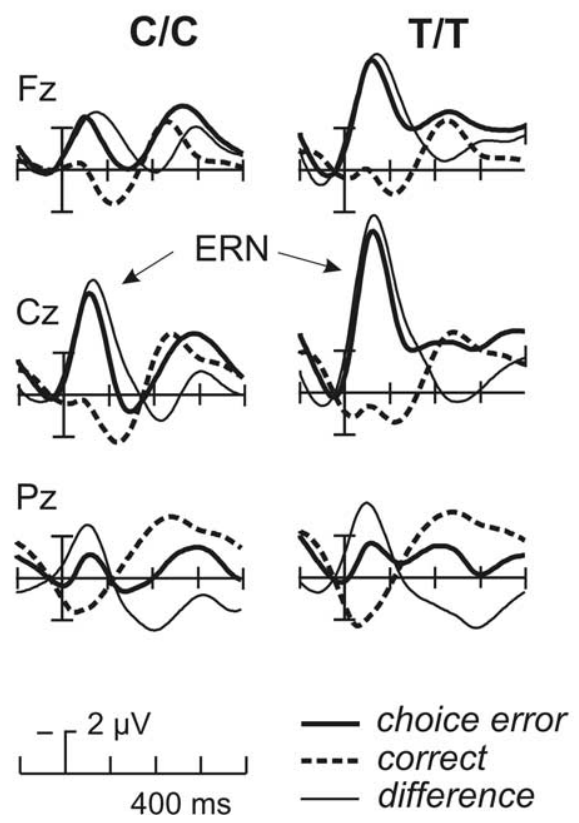

B

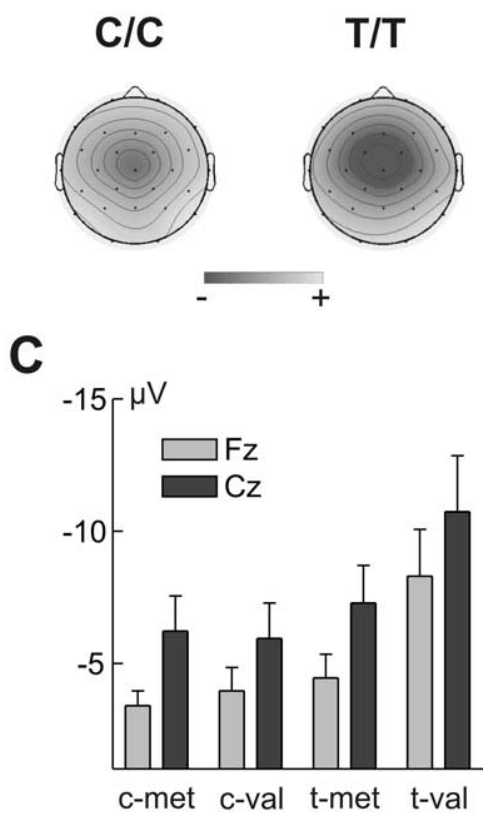

Figure 1. $\quad \boldsymbol{A}$, Grand averages of response-locked event-related potentials at midline electrodes in individuals homozygous for the C and Tallele (DRD4 -521). Depicted are ERPs for correct trials (dashed lines), error trials (solid thick lines) and the difference waveforms (solid thin lines). For illustration the data were bandpass filtered (bandpass 2-8 Hz). $\boldsymbol{B}$, Isovoltage maps of the difference waveform for the time window $0-100 \mathrm{~ms}$ in homozygotes for the $C$ and $T$ allele (maximum and minimum values in microvolts are -4.5 and 0.5 ). C, Mean trough-to-peak amplitudes at $\mathrm{Fz}$ and $\mathrm{Cz}$ for the four different groups with the respective SEs.

A
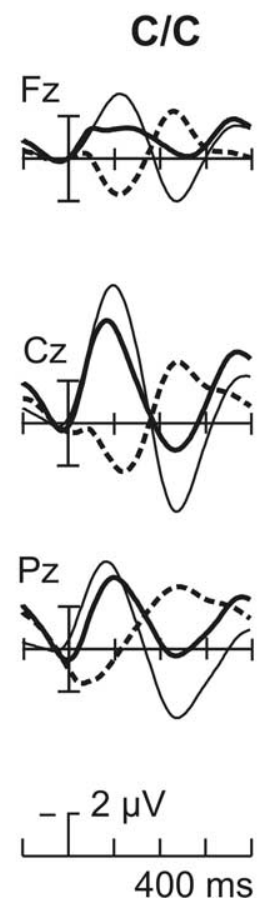
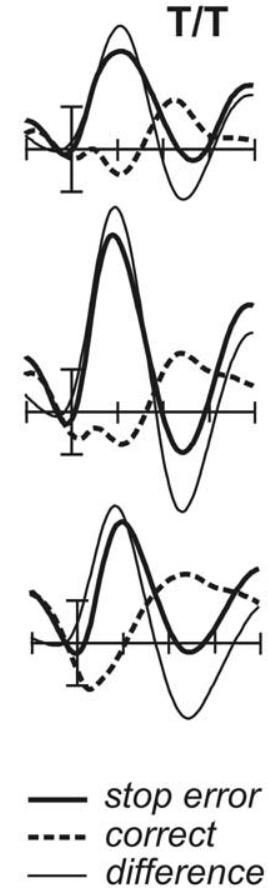

B

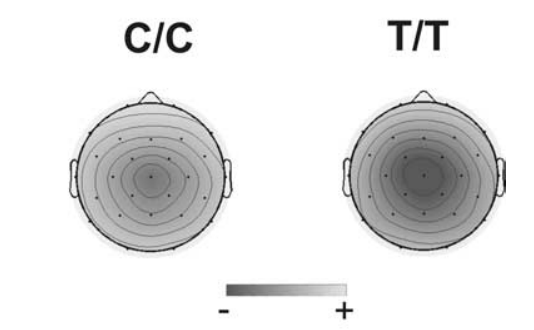

C

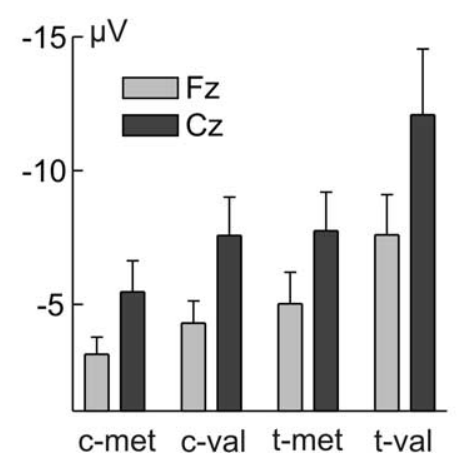

Figure 2. A, Grand averages of response-locked ERPs at midline electrodes in participants homozygous for the $C$ and $T$ allele (DRD4-521). Depicted are ERPs for correct trials (dashed lines), stop-error trials (solid thick lines), and the difference waveforms (solid thin lines). For illustration the data were bandpass filtered (bandpass filter, $2-8 \mathrm{~Hz}$ ). $\boldsymbol{B}$, Isovoltage maps of the difference waveform for the time window $0-130 \mathrm{~ms}$ in the homozygotes for the $C$ and $T$ allele (maximum and minimum values in microvolts are -5.5 and 0.5 ). C, Mean trough-to-peak amplitudes at $\mathrm{Fz}$ and $\mathrm{Cz}$ for the four different groups with the respective SEs.

suggested a larger ERN in Val and Thomozygous participants compared with $\mathrm{Met}$ and $C$ homozygotes. To investigate these genotype effects we subjected the mean amplitude at $\mathrm{Fz}$ and $\mathrm{Cz}$ between 0 and 100 $\mathrm{ms}$ for correct go trials and choice errors to a repeated measures ANOVA with the between-subject factors COMT and DRD4 and the within-subject factors condition (correct responses vs choice errors) and electrode $(\mathrm{Fz}$ and $\mathrm{Cz})$. We performed these analyses with bandpass-filtered data, in which the ERN is best detectable (bandpass $2-8 \mathrm{~Hz}$ ).

The differences in the choice ERN between the groups were reflected by a significant interaction of condition by DRD4 $\left(F_{(1,36)}=5.29, p=0.026\right)$ because of a higher ERN in $T$-homozygous (condition, $\left.F_{(1,19)}=38.96, p<0.001\right)$ compared with $C$-homozygous participants (condition, $F_{(1,19)}=32.97, p<0.001$ ) (Fig. $1 A, B$, for topography). We performed an additional through-to-peak measurement at $\mathrm{Fz}$ and $\mathrm{Cz}$ to confirm this genotype effect on the ERN. We defined the ERN peak as the most negative peak in the time-window between 0 and $130 \mathrm{~ms}$ and computed the amplitude difference to the preceding positive peak. The trough-to-peak amplitude at Fz and $\mathrm{Cz}$ for the four groups is depicted in Figure 1C. The corresponding ANOVA applied to this amplitude difference with the between-subject factors COMT and DRD4 and the between-subject factor electrode ( $\mathrm{Fz}$ and $\mathrm{Cz}$ ) confirmed again a main effect of DRD4 $\left(F_{(1,36)}=4.61, p=0.039\right)$.

Stop trials

Similarly to choice errors, an enhanced negativity peaking shortly after the false alarm responses in stop trials could be detected in the four groups. Visual inspection of the corresponding average waveforms suggested a larger negativity in $\mathrm{T}$ and $\mathrm{Val}$ participants compared with $C$ and Met participants (Fig. 2). As before we subjected the mean amplitude at $\mathrm{Fz}$ and $\mathrm{Cz}$ between 0 and $130 \mathrm{~ms}$ for correct go trials and stop errors to a repeated measures ANOVA with the between-subject factors COMT and DRD4 and the within-subject factors condition (correct go responses vs stop errors) and electrode (Fz and $\mathrm{Cz}$ ). We again used bandpass-filtered data for these analyses (bandpass 2-8 Hz).

Comparing stop errors with correct go responses revealed a significant effect for DRD4 (condition by DRD4, $F_{(1,36)}=6.75$, $p=0.013)$ and a marginal significant effect for COMT (condition by COMT, $F_{(1,36)}=$ 3.24, $p=0.080)$. The DRD4 effect was caused by a higher ERN in the 

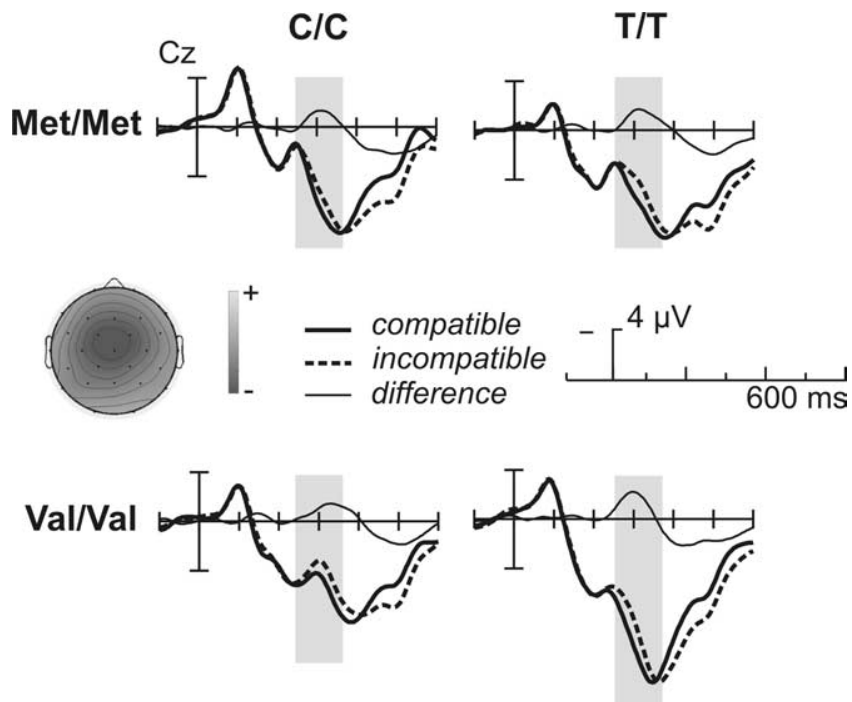

Figure 3. Grand averages of stimulus-locked ERPs at $\mathrm{C} z$ in the four groups. Depicted are compatible (solid thick line) and incompatible trials (dashed thick line) and the difference waveform (solid thin line). For illustration the data were low-pass filtered (low-pass filter, 12 $\mathrm{Hz}$ ). Shown is the isovoltage map of the difference waveform for the time window $250-350 \mathrm{~ms}$ (N2) in the complete sample (maximum and minimum values in microvolts are -1.0 and 0.5 ).

$T$-homozygous $\left(F_{(1,19)}=58.77, p<0.001\right)$ compared with $C$-homozygous participants $\left(F_{(1,19)}=49.07, p<0.001\right)$ (Fig. $2 A, B$, for topography). In parallel to the analyses of the choice ERN, we performed additionally a trough-to-peak amplitude at $\mathrm{Fz}$ and $\mathrm{Cz}$ to confirm these results for the stop negativity. The trough-to-peak amplitude at $\mathrm{Fz}$ and $\mathrm{Cz}$ in the four groups is shown in Figure 2C. We again found a significant effect for DRD4 $\left(F_{(1,36)}=4.85, p=0.034\right)$ and a tendency for $\operatorname{COMT}\left(F_{(1,36)}=\right.$ $3.50, p=0.069)$ (Fig. 2C) ${ }^{b}$

In summary, the results of the response-locked data showed a main effect of the DRD4 polymorphism on both the choice ERN and the stop-error negativity, with a tendency for an additional COMT effect on the stop-error negativity at least. It has to be noted, that the genotype effects were only small and the significance level not corrected for multiple comparisons. Future studies will be needed to replicate the current findings.

\section{ERPs: stimulus-locked data}

Incompatibility effects

Stimulus-locked ERPs in both compatible and incompatible go trials were characterized by a typical visual N1-P2 complex, followed by a frontocentral negativity peaking at $\sim 300 \mathrm{~ms}$ (N2), that was superimposed on an extended positivity with a posterior maximum (P300). As expected, the N2 was enhanced for incompatible compared with compatible trials. Moreover, the peak of the P300 component was delayed in incompatible trials (Fig. 3).

To probe the N2 incompatibility effect for group differences, we subjected the mean amplitude between 250 and 350 $\mathrm{ms}$ at midline electrodes to a repeated measures ANOVA with the between-subjects factors COMT and DRD4 and the within-subject factors condition (compatible vs incompatible) and electrode (levels: $\mathrm{Fz}, \mathrm{Cz}$, and $\mathrm{Pz}$ ). These analyses were

${ }^{b}$ The analyses for the error-related negativity were additionally performed in unfiltered data. Both the DRD4 effect on the choice ERN (mean amplitude at $\mathrm{Fz}, 0-100 \mathrm{~ms}$ ) and stop negativity (mean amplitude at $\mathrm{Fz}, 0-130 \mathrm{~ms}$ ) were confirmed (both $p<0.05$ ).

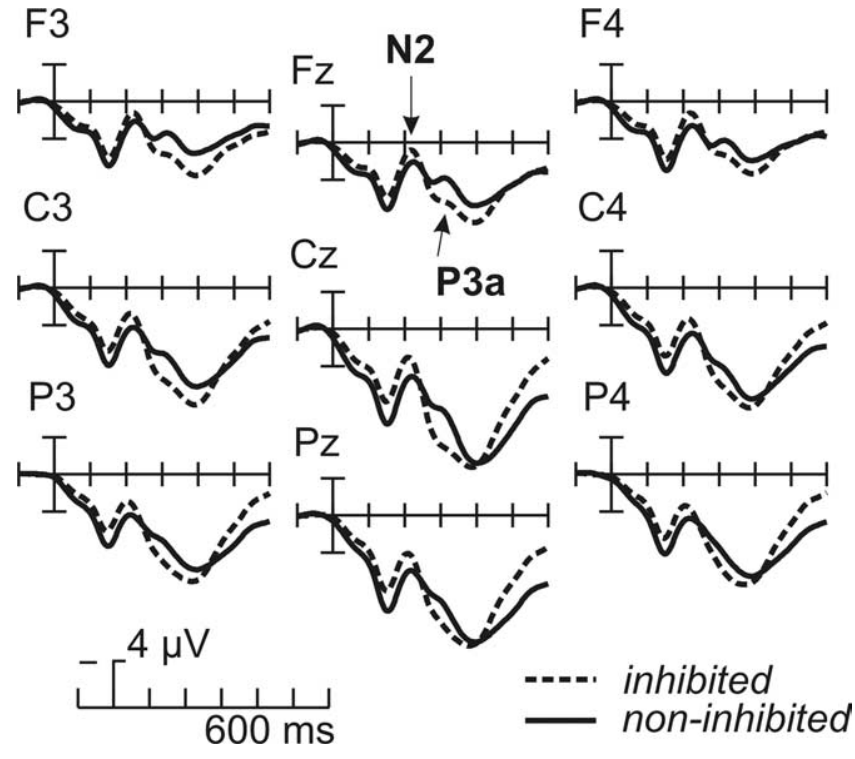

Figure 4. Grand averages of stop stimulus-locked ERPs at selected electrodes for the complete sample. Depicted are inhibited (dashed lines) and noninhibited trials (solid lines). Again, the data were low-pass filtered for illustration $(12 \mathrm{~Hz})$ and N2 and P3a are highlighted.

performed with unfiltered data. The incompatibility effect on the N2 amplitude was confirmed by the interaction of condition by electrode $\left(F_{(1,36)}=6.86, p=0.003\right)$. Yet, neither an interaction of COMT or DRD4 and condition nor main effects of genotypes on the N2 incompatibility effect were detected (all $p$ values $>0.1$ ), suggesting that both polymorphisms had no effect on this neurophysiological marker of conflict monitoring.

To tap into possible genotype effects on the P300 latency, we subjected the P300 peak latency at $\mathrm{Pz}$ (most positive peak between 300 and $600 \mathrm{~ms}$ ) to a repeated measures ANOVA with the same factors as before (except electrode). The finding of a delayed P300 in incompatible compared with compatible trials was reflected by the significant main effect of condition $\left(F_{(1,36)}=18.98\right.$, $p<0.001)$. No interaction of COMT and DRD4 with condition was detected (all $p$ values $>0.1$ ). Thus, both incompatibility effects (N2 amplitude and P3 latency) showed no modulation by the dopaminergic polymorphisms. There was a slight tendency for a generally enhanced P300 amplitude (defined as the mean amplitude between 300 and $600 \mathrm{~ms}$ ) in the $T / T$-group, indicated by a marginal significant main effect of DRD $4\left(F_{(1,36)}=3.33, p=\right.$ 0.076).

\section{Inhibitory effects}

ERPs time-locked to the stop signal showed a sharp central negativity at $\sim 200 \mathrm{~ms}(\mathrm{~N} 2)^{c}$ followed by a broad posterior positivity (Fig. 4). This negativity was clearly detectable in both inhibited and noninhibited trials. In trials of failed inhibitions a second negativity followed, peaking at $\sim 300 \mathrm{~ms}$, reflecting the stoperror-related negativity which was previously analyzed in the section on response-locked ERPs. This enhanced negativity for failed inhibitions was overlapping with the previously reported stop-related P3a, that was shown to be larger for successful inhibitions (Liotti et al., 2005).

When directly contrasting trials of successful inhibitions in 
A

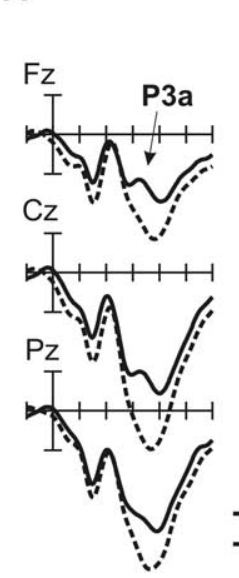

B

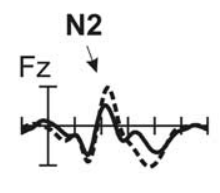

bandpass filtered $(2-12 \mathrm{~Hz})$

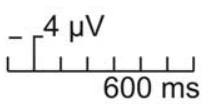

Val: inhibited

Met: inhibited
C

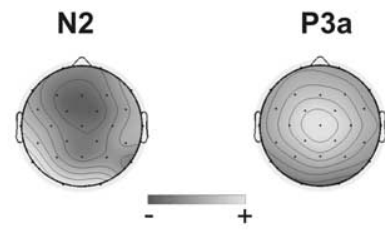

D

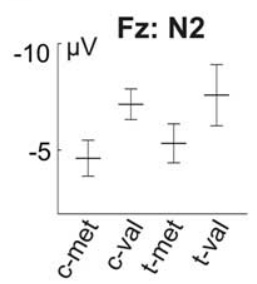

Figure 5. A, Grand averages of stop stimulus-locked ERPs at midline electrodes in the Val (dashed lines) and Met groups (solid lines) for successfully inhibited trials. The P3a is indicated with an arrow. $\boldsymbol{B}$, For illustration of the N2 effect, ERPs in inhibited trials are shown for the two groups at the Fz electrode with a bandpass filter of 2-12 Hz. C, Depicted are the isovoltage maps of the amplitude difference between Val/Val and Met/Met for the stop N2 (200-220 ms; left) and the P3a (300-350 ms; right). As the groups differed in the preceding positivity of the N2, we show for illustration the amplitude difference relative to the baseline from 100 to $200 \mathrm{~ms}$. Maximum and minimum values are for the $\mathrm{N} 2$ are -1.2 and $0.2 \mu \mathrm{V}$, and for the P3a are -1.5 and $5.5 \mu \mathrm{V}$. D. Mean peak-to-peak amplitudes for the $\mathrm{N} 2$ at Fz for the four different groups in inhibited trials (with their respective SEs). $\boldsymbol{E}$, Mean amplitudes for the P3a in the four different groups in inhibited trials (300-350 ms) with their respective SE.

the different groups, an increased amplitude of the stop N2 and an enhanced positivity was evident in the Val participants compared with Met participants (Fig. 5; note that for illustration purposes, a bandpass filter of $2-12 \mathrm{~Hz}$ was used in Fig. $5 B$ ). We defined the stop N2 as the peak-to-peak amplitude (difference between the negative peak $180-250 \mathrm{~ms}$ and the preceding positive peak; determined in low-pass filtered data, low-pass $12 \mathrm{~Hz}$ ) to avoid a confound with differences in the underlying positivity. The peak-to-peak amplitude was measured at Fz, where the stop $\mathrm{N} 2$ and the group differences were largest and is depicted for the four groups in Figure 5D (Fig. 5C, for scalp topography). The larger stop N2 in the Val group was confirmed by the main effect of COMT $\left(F_{(1,36)}=5.59, p=0.024 ; \mathrm{Val},-7.37 \mu \mathrm{V} ; \mathrm{Met},-4.96\right.$ $\mu \mathrm{V})$. We observed no main effect of or interaction with DRD4 $(p>0.1)$ (Fig. 5D).

After the stop N2, an extended positivity (P3a) could be detected (Fig. 5A,C, for scalp topography), peaking at $\sim 300-350$ $\mathrm{ms}$ and maximally pronounced at central electrodes (Liotti et al., 2005). The inhibition-related P3a was similar to the stop N2 higher in Val than in Met carriers. This was corroborated by a mean amplitude measurement at midline electrodes between 300 and $350 \mathrm{~ms}$ (main effect COMT: $F_{(1,36)}=4.48, p=0.041$ ). The mean amplitude in the four groups is depicted in Figure $5 E$.

\section{Discussion}

The present study demonstrates the impact of two polymorphisms in the dopaminergic system on neurophysiological markers of executive functions. Genetic variability in the DRD4-521 polymorphism predicted interindividual differences in error-related prefrontal activity and in behavioral post-error adjustments. Additionally, a genetically driven lower level of COMT was associated with reduced inhibitionrelated frontal activity.

\section{Error monitoring}

DRD4-521 $T$ allele-homozygous participants showed increased frontocentral negativities after both choice and stop errors com-

E

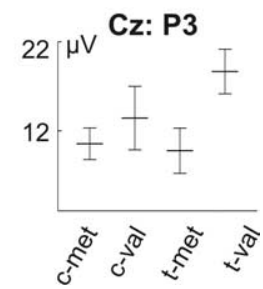

pared with $C$ allele carriers. Moreover, a tendency for an additional COMT effect was seen with a larger negativity in $\mathrm{Val}$ compared with Met-homozygous participants for stop errors. These results hint at specific effects on action monitoring processes reflected in the error negativity. On a behavioral level, $T$-homozygous participants showed greater posterror adjustments in particular after failed inhibitions.

Different models have been suggested regarding the ERN, relating it to, for example, error detection (Gehring et al., 1993) or conflict monitoring (Yeung et al., 2004). Holroyd and Coles (2002) previously proposed a direct link of the ERN to activity in the mesencephalic dopaminergic system. Their reinforcement-learning model assumes a response-monitoring system in the basal ganglia, producing positive and negative prediction error signals, that are coded as phasic increases or decreases of activity of mesencephalic dopaminergic neurons. The ERN emerges, when phasic decreases in the activity of mesencephalic dopaminergic neurons disinhibit the apical dendrites in motor neurons in the ACC. Finally the ACC and other areas as the amygdala or hippocampus, which receive input from mesencephalic dopaminergic neurons, project back to the basal ganglia, where the information is used to improve prediction of the monitoring system.

Different mechanisms are possible how D4 receptor availability and COMT level might affect functioning in this loop. The dopamine D4 receptor is expressed mainly in the prefrontal cortex (including ACC), in the amygdala and in the hippocampus, making a modulatory impact in these areas very likely (Oak et al., 2000). Moreover, studies with D4 receptor knock-out mice suggest that D4 receptor expression rate leads to compensatory changes in mesencephalic dopamine production, resulting in different tonic dopamine levels in participants homozygous for the $T$ and $C$ allele in DRD4 (Rubinstein et al., 1997). Thus, the DRD4 polymorphism in question might also directly impact prediction error signaling in the basal ganglia and thereby the ERN. Interestingly, evidence exists for an association with schizophrenia and ADHD for DRD4-521 alleles, supposedly stemming from altered prefrontal activity (Okuyama et al., 1999; Mill et al., 2003; Xing et al., 2003). Additionally, several studies demonstrated disturbed performance monitoring mechanisms reflected in a reduced ERN in schizophrenic and ADHD patients (Kopp and Rist, 1999; Alain et al., 2002; Mathalon et al., 2002; Liotti et al., 2005). Although the direct impact of the SNP-521 on D4 receptor availability remains controversial (Okuyama et al., 1999; Kereszturi et al., 2006), the present study provides direct evidence for differences in specific prefrontal functions driven by this polymorphism, that might contribute to a higher risk for psychiatric disorders as schizophrenia or ADHD.

COMT is the main mechanism for prefrontal dopamine turnover because of a sparse expression of the dopamine transporter (Matsumoto et al., 2003; Chen et al., 2004). In addition to the direct impact COMT might thus have on prefrontal functioning, different mechanisms have been pro- 
posed for a relationship between COMT activity and dopamine release in the basal ganglia: extrasynaptic COMT in the basal ganglia might directly influence local tonic dopaminergic activity (Huotari et al., 2002) or, alternatively, prefrontal COMT level might drive striatal dopamine levels via a regulation of glutamatergic projections from PFC to the basal ganglia (Bilder et al., 2004; Meyer-Lindenberg et al., 2006). More specifically, lower COMT level will increase firing of pyramidal neurons in the PFC and increase corticostriatal glutamate transmission, resulting finally in increased glutamatestimulated tonic dopamine release in the striatum and suppression of phasic dopamine release (Bilder et al., 2004). The lower COMT level in individuals homozygous for the Met allele is thus supposed to be associated with an increased tonic dopamine release and reduced phasic dopamine responses in the basal ganglia (Yacubian et al., 2007), which leads to the prediction of a reduced ERN which is what we found.

\section{Conflict monitoring}

Regarding the incompatibility effects in the stimulus-locked data, we observed the typical enhanced negativity for incompatible compared with compatible trials and a delayed P300. However, no differentiation between the groups could be detected in these markers of conflict monitoring. This is somehow surprising, because Fossella et al. (2002) reported a COMT polymorphism impact on the incompatibility effect even on the behavioral level, with the Met participants showing a tendency for a higher distractability and thereby higher incompatibility effect. Moreover, a previous functional magnetic resonance imaging study reported an effect for COMT on ACC activity during a similar, albeit more demanding flanker task (Blasi et al., 2005). Blasi et al. (2005) demonstrated a linear relationship of ACC activity driven by attentional control with the number of $\mathrm{Val}$ alleles, with the highest ACC activity in homozygous Val participants. Because Val participants at the same time exhibited the poorest performance, the authors conclude a less efficient prefrontal processing in these participants. However, this effect was most prominent at the highest level of attentional control, suggesting that the null effects in the present study might be caused by an overall lower demand for conflict monitoring. It should be pointed out though, that only the participants homozygous for the $\mathrm{C} / \mathrm{C}$ and $\mathrm{Val} / \mathrm{Val}$ alleles showed unspecific performance deficits in the present study (overall slower reaction times), but no increased error rates could be detected in Vall Val homozygotes as in the study of Blasi et al. (2005). In fact, there was even a tendency for higher error rates in Met/Met participants, although this was not significant.

\section{Response inhibition}

Stimulus-locked data for the stop signal showed a typical inhibition-related stop N2, that was observable in both successfully inhibited trials and failed inhibitions. This was followed by a positive deflection (P3), that was at frontal sites more pronounced for successfully inhibited trials.

The stop N2 in stop signal tasks has been suggested to reflect monitoring processes in the prefrontal cortex, related to a "red flag" signal triggering the inhibitory process (Kok, 1986). We observed an effect of the COMT polymorphism on the stop N2, such that Val participants revealed a greater amplitude compared with Met carriers. This goes in line with previous evidence, pointing at interindividual differences in prefrontal processes driven by genetically determined dopa- minergic variability (Egan et al., 2001; Blasi et al., 2005; Schott et al., 2006). Interestingly, similar group differences in the stop N2 have been observed comparing boys with ADHD and a healthy control group (Pliszka et al., 2000). The N2 was shown to be greatly reduced in ADHD children, pointing at a neurophysiological correlate of their impaired inhibitory control. Given the previously reported link between the COMT polymorphism and ADHD (Bellgrove et al., 2005b), the present data thus suggest a possible underlying neural mechanism for this relationship. Because the DRD4 polymorphism has been also associated with $\mathrm{ADHD}$, it remains an open question why we did not observe any effects related to DRD4.

Additionally, participants homozygous for the Val allele (COMT) presented an enhanced inhibition-related central positivity (also referred to as P3a) compared with Methomozygote participants (Kok et al., 2004; Bekker et al., 2005; Liotti et al., 2005). As the time needed to successfully inhibit a response, indicated by the SSRT, finishes clearly before the P3, this component cannot reflect the inhibition process per se. It has rather been related to a later stage of the inhibitory process, indexing the monitoring of its successful implementation instigated by the anterior cingulate or pre-SMA (Liotti et al., 2005). Similarly to the stop N2, the P3a has been shown to be diminished in ADHD and related to behavioral indices of response inhibition (Liotti et al., 2005). Interestingly, at least in the homozygotes for the $\mathrm{Val}$ allele and the $\mathrm{T}$ allele, the increased P3a was associated with a shorter SSRT (i.e., better task performance). This is in contrast to previous studies related to working memory and attentional control (Egan et al., 2001; Blasi et al., 2005), demonstrating genetically driven increased prefrontal activity associated with impaired task performance.

\section{Conclusions}

The present study is the first to provide direct evidence for the impact of two dopaminergic polymorphisms on neurophysiological correlates of performance monitoring by means of ERPs (for data on serotonin-related genetic influence on the ERN, see Fallgatter et al., 2004). The results suggest a distinct effect of the DRD4 SNP-521 on error monitoring, evident in the group differences in the choice ERN and the stop-related ERN. Moreover, increased post-error slowing in the participants homozygous for the $T$ allele (DRD4) hint at behavioral costs of this elevated performance monitoring. However, the COMT Val108/158Met polymorphism affected inhibitionrelated neurophysiological markers, with the participants carrying the $\mathrm{Val}$ allele revealing enhanced prefrontal processing. Interestingly, especially for the "extreme" group of participants homozygous for T allele in DRD4 and the Val allele in COMT, the present study demonstrates increased prefrontal functioning related to performance monitoring processes that were associated with both behavioral costs, evident in the increased post-error slowing, and behavioral benefits, observable in the shorter SSRT. This parallels previous observations suggesting distinct behavioral costs emanating from elevated or diminished dopaminergic functioning (Cohen et al., 2002; Bilder et al., 2004; Seamans and Yang, 2004).

\section{References}

Akil M, Kolachana BS, Rothmond DA, Hyde TM, Weinberger DR, Kleinman JE (2003) Catechol-O-methyltransferase genotype and dopamine regulation in the human brain. J Neurosci 23:2008-2013.

Alain C, McNeely HE, He Y, Christensen BK, West R (2002) Neurophysio- 
logical evidence of error-monitoring deficits in patients with schizophrenia. Cereb Cortex 12:840-846.

Band GP, van Boxtel GJ (1999) Inhibitory motor control in stop paradigms: review and reinterpretation of neural mechanisms. Acta Psychol (Amst) 101:179-211.

Band GP, van der Molen MW, Logan GD (2003) Horse-race model simulations of the stop-signal procedure. Acta Psychol (Amst) 112:105-142.

Barr CL (2001) Genetics of childhood disorders: XXII. ADHD, Part 6: The dopamine D4 receptor gene. J Am Acad Child Adolesc Psychiatry 40:118-121.

Bekker EM, Kenemans JL, Hoeksma MR, Talsma D, Verbaten MN (2005) The pure electrophysiology of stopping. Int J Psychophysiol 55:191-198.

Bellgrove MA, Hawi Z, Lowe N, Kirley A, Robertson IH, Gill M (2005a) DRD4 gene variants and sustained attention in attention deficit hyperactivity disorder (ADHD): effects of associated alleles at the VNTR and -521 SNP. Am J Med Genet B Neuropsychiatr Genet 136:81-86.

Bellgrove MA, Domschke K, Hawi Z, Kirley A, Mullins C, Robertson IH, Gill M (2005b) The methionine allele of the COMT polymorphism impairs prefrontal cognition in children and adolescents with ADHD. Exp Brain Res 163:352-360.

Bilder RM, Volavka J, Lachman HM, Grace AA (2004) The catechol-Omethyltransferase polymorphism: relations to the tonic-phasic dopamine hypothesis and neuropsychiatric phenotypes. Neuropsychopharmacology 29:1943-1961.

Blasi G, Mattay VS, Bertolino A, Elvevag B, Callicott JH, Das S, Kolachana BS, Egan MF, Goldberg TE, Weinberger DR (2005) Effect of catechol-Omethyltransferase val158met genotype on attentional control. J Neurosci 25:5038-5045.

Bonfield J, Beal K, Cheng Y, Jordan M, Staden R (1995) Staden package. Retrieved December 5, 2007, from http://staden.sourceforge.net/.

Carter CS, Braver TS, Barch DM, Botvinick MM, Noll D, Cohen JD (1998) Anterior cingulate cortex, error detection, and the online monitoring of performance. Science 280:747-749.

Chen J, Lipska BK, Halim N, Ma QD, Matsumoto M, Melhem S, Kolachana BS, Hyde TM, Herman MM, Apud J, Egan MF, Kleinman JE, Weinberger DR (2004) Functional analysis of genetic variation in catechol-O-methyltransferase (COMT): effects on mRNA, protein, and enzyme activity in postmortem human brain. Am J Hum Genet 75:807-821.

Cohen JD, Braver TS, Brown JW (2002) Computational perspectives on dopamine function in prefrontal cortex. Curr Opin Neurobiol 12:223-229.

Diaz-Asper CM, Weinberger DR, Goldberg TE (2006) Catechol-Omethyltransferase polymorphisms and some implications for cognitive therapeutics. NeuroRx 3:97-105.

Egan MF, Goldberg TE, Kolachana BS, Callicott JH, Mazzanti CM, Straub RE, Goldman D, Weinberger DR (2001) Effect of COMT Val108/158 Met genotype on frontal lobe function and risk for schizophrenia. Proc Natl Acad Sci USA 98:6917-6922.

Eriksen BA, Eriksen CW (1974) Effects of noise letters upon the identification of a target letter in a nonsearch task. Percept Psychophys 16:143.

Falkenstein M, Hohnsbein J, Hoormann J, Blanke L (1990) Effects of errors in choice reaction tasks on the ERP under focused and divided attention. In: Psychophysiological brain research (Brunia CH, Gaillard AW, Kok A, eds), pp 192-195. Tilburg, The Netherlands: Tilburg UP.

Falkenstein M, Hielscher H, Dziobek I, Schwarzenau P, Hoormann J, Sunderman B, Hohnsbein J (2001) Action monitoring, error detection, and the basal ganglia: an ERP study. NeuroReport 12:157-161.

Fallgatter AJ, Herrmann MJ, Roemmler J, Ehlis AC, Wagener A, Heidrich A, Ortega G, Zeng Y, Lesch KP (2004) Allelic variation of serotonin transporter function modulates the brain electrical response for error processing. Neuropsychopharmacology 29:1506-1511.

Fossella J, Sommer T, Fan J, Wu Y, Swanson JM, Pfaff DW, Posner MI (2002) Assessing the molecular genetics of attention networks. BMC Neurosci 3:14.

Gehring WJ, Knight RT (2000) Prefrontal-cingulate interactions in action monitoring. Nat Neurosci 3:516-520.

Gehring WJ, Goss B, Coles MG, Meyer DE (1993) A neural system for error detection and compensation. Psychol Sci 4:385-390.

Holroyd CB, Coles MG (2002) The neural basis of human error processing: reinforcement learning, dopamine, and the error-related negativity. Psychol Rev 109:679-709.

Huotari M, Santha M, Lucas LR, Karayiorgou M, Gogos JA, Mannisto PT (2002) Effect of dopamine uptake inhibition on brain catecholamine levels and locomotion in catechol-O-methyltransferase-disrupted mice. J Pharmacol Exp Ther 303:1309-1316.

Huynh H, Feldt LS (1976) Estimation of the box correction for degrees of freedom from sample data in randomized block and splitsplot designs. J Educ Statist 1:69-82.

Kereszturi E, Kiraly O, Barta C, Molnar N, Sasvari-Szekely M, Csapo Z (2006) No direct effect of the $-521 \mathrm{C} / \mathrm{T}$ polymorphism in the human dopamine D4 receptor gene promoter on transcriptional activity. BMC Mol Biol 7:18.

Kok A (1986) Effects of degradation of visual stimulation on components of the event-related potential (ERP) in go/nogo reaction tasks. Biol Psychol 23:21-38.

Kok A, Ramautar JR, De Ruiter MB, Band GPH, Ridderinkof KR (2004) ERP components associated with successful and unsuccessful stopping in a stop-signal task. Psychophysiology 41:9-20.

Kopp B, Rist F (1999) An event-related brain potential substrate of disturbed response monitoring in paranoid schizophrenic patients. J Abnorm Psychol 108:337-346.

Lachman HM, Morrow B, Shprintzen R, Veit S, Parsia SS, Faedda G, Goldberg R, Kucherlapati R, Papolos DF (1996) Association of codon 108/ 158 catechol-O-methyltransferase gene polymorphism with the psychiatric manifestations of velo-cardio-facial syndrome. Am J Med Genet $67: 468-472$.

Laurens KR, Ngan ETC, Bates AT, Kiehl KA, Liddle PF (2003) Rostral anterior cingulate cortex dysfunction during error processing in schizophrenia. Brain 126:610-622.

Liotti M, Pliszka SR, Perez R, Kothmann D, Woldorff MG (2005) Abnormal brain activity related to performance monitoring and error detection in children with ADHD. Cortex 41:377-388.

Mathalon DH, Fedor M, Faustman WO, Gray M, Askari N, Ford JM (2002) Response-monitoring dysfunction in schizophrenia: an event-related brain potential study. J Abnorm Psychol 111:22-41.

Matsumoto M, Weickert CS, Akil M, Lipska BK, Hyde TM, Herman MM, Kleinman JE, Weinberger DR (2003) Catechol O-methyltransferase mRNA expression in human and rat brain: evidence for a role in cortical neuronal function. Neuroscience 116:127-137.

Meyer-Lindenberg A, Kohn PD, Kolachana B, Kippenhan S, McInerney-Leo A, Nussbaum R, Weinberger DR, Berman KF (2005) Midbrain dopamine and prefrontal function in humans: interaction and modulation by COMT genotype. Nat Neurosci 8:594-596.

Meyer-Lindenberg A, Nichols T, Callicott JH, Ding J, Kolachana B, Buckholtz J, Mattay VS, Egan M, Weinberger DR (2006) Impact of complex genetic variation in COMT on human brain function. Mol Psychiatry 11:867-877.

Mill J, Fisher N, Curran S, Richards S, Taylor E, Asherson P (2003) Polymorphisms in the dopamine D4 receptor gene and attention-deficit hyperactivity disorder. NeuroReport 14:1463-1466.

Mitsuyasu H, Kawasaki H, Ninomiya H, Kinukawa N, Yamanaka T, Tahira T, Stanton JVP, Springett GM, Hayashi K, Tashiro N, Kanba S (2007) Genetic structure of the dopamine receptor D4 gene (DRD4) and lack of association with schizophrenia in Japanese patients. J Psychiatr Res 41:763-775.

Oak JN, Oldenhof J, Van Tol HH (2000) The dopamine D(4) receptor: one decade of research. Eur J Pharmacol 405:303-327.

Okuyama Y, Ishiguro H, Toru M, Arinami T (1999) A genetic polymorphism in the promoter region of DRD4 associated with expression and schizophrenia. Biochem Biophys Res Commun 258:292-295.

Pliszka SR, Liotti M, Woldorff MG (2000) Inhibitory control in children with attention-deficit/hyperactivity disorder: event-related potentials identify the processing component and timing of an impaired rightfrontal response-inhibition mechanism. Biol Psychiatry 48:238-246.

Rabbitt PM (1966) Errors and error correction in choice-response tasks. J Exp Psychol 71:264-272.

Rodriguez-Fornells A, Kurzbuch AR, Münte TF (2002) Time course of error detection and correction in humans: neurophysiological evidence. J Neurosci 22:9990-9996.

Rubinstein M, Phillips TJ, Bunzow JR, Falzone TL, Dziewczapolski G, Zhang G, Fang Y, Larson JL, McDougall JA, Chester JA, Saez C, Pugsley TA, 
Gershanik O, Low MJ, Grandy DK (1997) Mice lacking dopamine D4 receptors are supersensitive to ethanol, cocaine, and methamphetamine. Cell 90:991-1001.

Schott BH, Seidenbecher CI, Fenker DB, Lauer CJ, Bunzeck N, Bernstein HG, Tischmeyer W, Gundelfinger ED, Heinze HJ, Duzel E (2006) The dopaminergic midbrain participates in human episodic memory formation: evidence from genetic imaging. J Neurosci 26:1407-1417.

Seamans JK, Yang CR (2004) The principal features and mechanisms of dopamine modulation in the prefrontal cortex. Prog Neurobiol 74:1-58.

Ullsperger M, von Cramon DY (2001) Subprocesses of performance monitoring: a dissociation of error processing and response competi- tion revealed by event-related fMRI and ERPs. Neuroimage 14:1387-1401.

Xing Q, Wu S, Lin Z, Li H, Yang J, Feng G, Wang M, Yang W, He L (2003) Association analysis of polymorphisms in the upstream region of the human dopamine D4 receptor gene in schizophrenia. Schizophr Res 65:9-14.

Yacubian J, Sommer T, Schroeder K, Glascher J, Kalisch R, Leuenberger B, Braus DF, Buchel C (2007) Gene-gene interaction associated with neural reward sensitivity. Proc Natl Acad Sci USA 104:8125-8130.

Yeung N, Cohen JD, Botvinick MM (2004) The neural basis of error detection: conflict monitoring and the error-related negativity. Psychol Rev 111:931-959. 